\title{
Pupillary diameter and reaction to light in preterm neonates
}

\author{
J Robinson, A R Fielder
}

\begin{abstract}
The pupil controls the amount of light entering the eye. We have examined both the time course for the development of the light reflex and pupil diameter before and after this event in 50 neonates. The pupillary light reflex was absent in all neonates of less than $\mathbf{3 0}$ weeks' gestational age, it gradually developed from this age and by 35 weeks it was present in all. Several possible mechanisms that may explain the onset of this reflex are considered. Before the onset of the pupil reflex mean horizontal pupillary diameter was $3.46 \mathrm{~mm}$ compared with $3.02 \mathrm{~mm}$ after the reflex development. We have estimated the amount of light reaching the retina (retinal irradiance) for preterm neonates and although it is less than that known to cause retinal damage in animals, further research is necessary to determine whether early light exposure has an adverse or beneficial effect on the immature visual system.
\end{abstract}

The pupil regulates the amount of light entering the eye by reflex dilatation or constriction in response to changes in the illumination. Few studies have been performed that have explored the development of the light reflex in neonates. In 1966 Robinson investigated the development of 20 types of reflexes in an attempt to produce an assessment that would be useful to the paediatrician interested in distinguishing between preterm and small for date neonates. ${ }^{1}$ Of the reflexes examined he found that the presence of the pupillary light reflex was one of five that were highly correlated with gestational age. Finnström designed a study to assess the use of reflex testing as part of a gestational age assessment. $^{2}$ Babies of less than 29 weeks' gestational age $(n=4)$ had no pupillary response to light, but by 31 weeks' gestational age the reflex appeared to be present, although sluggish in all. When the presence of the pupillary light reflex was examined as a function of gestation, he found a correlation coefficient of only $0 \cdot 24$, although he accepted that this may have been due to the small number $(n=6)$ of infants of less than 32 weeks' gestation in this study. In both of these studies the numbers of babies of less than 29 weeks' gestational age were small and their gestational ages were not given. This indicates the need to examine the pupil of the very immature infant: to date the pupil diameter of the preterm neonate has been mentioned on two occasions only and divergent values of 1.5 $\mathrm{mm}^{3}$ and $2.9 \mathrm{~mm}^{4}$ have been quoted. We therefore decided to study pupillary diameter and light reflex prospectively, and we report, first, the time course of the development of the pupillary light reflex and second,pupil diameter in infants with and without pupillary constriction to light.

\section{Subjects and methods}

SUBJECTS

Preterm babies with gestational age less than 37 weeks (WHO classification) admitted to the neonatal unit of the Leicester Royal Infirmary between February and July 1988 were enrolled in the investigation of pupillary diameter. Those who had no light reflex at the first examination were enrolled in the longitudinal study of reflex development. But for the study of pupil diameter, infants with a light reflex on the initial examination were also included. Postmenstrual age was defined as the gestational age, as assessed by the paediatrician at birth, plus the postnatal age. Ethical approval for the study was granted by the Leicestershire Area Health Authority. Full details of the infants are included in the table.

\section{APPARATUS}

The Medical-Nikkor Auto $200 \mathrm{~mm} \mathrm{f} / 5.6$ lens was designed for taking close up photographs of known reproduction ratio of $1: 15$, use of an auxillary lens can alter this ratio to $1: 1$. As the lens is fixed-focus, focusing is achieved simply by pointing the camera at the eye and moving it back and forth until the eye is in sharp focus. The lens to subject distance was $221 \mathrm{~mm}$ using this combination of lenses and the subject field was 24 by $36 \mathrm{~mm}$.

The lens was attached to a Nikkon FE camera back, and the power for the ring flash was produced by the supplied AC converter. The flash illumination was reduced to one fourth of

Details of subjects studied

\begin{tabular}{|c|c|c|c|}
\hline \multirow{2}{*}{$\begin{array}{l}\text { Gestational } \\
\text { (weeks) }\end{array}$} & \multicolumn{2}{|c|}{ No of infants } & \multirow{2}{*}{$\begin{array}{l}\text { Median (range) } \\
\text { birth weight (g) }\end{array}$} \\
\hline & Total & $\begin{array}{l}\text { With pupils } \\
\text { reacting to } \\
\text { light }\end{array}$ & \\
\hline $\begin{array}{r}26 \\
27 \\
28 \\
29 \\
30 \\
31 \\
32 \\
33 \\
34 \\
>34\end{array}$ & $\begin{array}{l}4 \\
6 \\
4 \\
6 \\
4 \\
4 \\
4 \\
6 \\
6 \\
4 \\
6\end{array}$ & $\begin{array}{l}0 \\
1^{*} \\
0 \\
0 \\
2 \\
4 \\
6 \\
5 \\
2 \\
6\end{array}$ & $\begin{aligned} 790 & (760-820) \\
750 & (760-880) \\
855 & (650-980) \\
1045 & (1030-1060) \\
1095 & (1030-1420) \\
1370 & (1020-2121) \\
1570 & (1520-1620) \\
1645 & (1580-1700) \\
1750 & (1680-2200) \\
1990 & (1520-2500)\end{aligned}$ \\
\hline
\end{tabular}

*In this infant a slight, slow reaction to light was noted in the first examination. However, no response to light was noted in either of the next two examinations, made at weekly intervals. 
its full value at higher reproduction ratios. The output of the ring flash was filtered using a combination of EE and $30 \mathrm{CC}$ Blue filters (Kodak Wratten) which effectively blocks $99 \%$ of the ultraviolet light emitted by the flash. A flash may be used as the latency of the pupillary light reflex is always longer than the duration of the film exposure, thus the photograph will accurately record the size of the non-constricted pupil..$^{5}$

Fast film (Kodak TMX Professional, ASA 400) was loaded to reduce the duration of exposure to a minimum. An aperture of $f 32$ was used, which in combination with the $\times 1$ auxillary lens produces a depth of field of \pm 1.86 $\mathrm{mm}$, thus the eye was only in focus when the lens to eye distance was in the range $219 \cdot 14$ to $222.86 \mathrm{~mm}$. Similarly, the lens system could only produce clear images of the pupil if there was a $90^{\circ}$ angle between the iris plane and the lens thus the camera must be 'on-axis' (with a tolerance of $\pm 2^{\circ}$ ).

\section{PROTOCOL}

The babies were examined at weekly intervals while on the neonatal unit commencing in all during the first week of life. Eyelids were opened manually or in the case of very immature infants an eyelid speculum was used after instillation of topical anaesthesia oxybuprocaine hydrochloride $0 \cdot 4 \%$.

Photographs were taken usually first of the left eye; the presence of the pupillary reflex tested using the light from a direct ophthalmoscope $(6 \mathrm{~W})$ for a minimum duration of five seconds. Iris colour was noted. Similar records were then made for the right eye after a minimum five minute interval.

\section{ANALYSIS OF RESULTS}

Horizontal and vertical pupil diameters were measured directly from the film negatives using a magnified graticule scale. Any image in which either the pupil was not clearly visible or the image of the ring flash was not in the centre of the pupil were discarded. Diameters were measured both by a naive observer and by one of the experimenters with good interobserver agreement being noted $(r=0 \cdot 93)$.

\section{STATISTICAL ANALYSIS}

Light reflex: methods of analysis and presentation The results were grouped into six postmenstrual age categories. In the first category $(<27$ weeks), the pupil response to light was invariably absent while in the last category ( $>34$ weeks) this was always present. For each period responses were included if the infant was born in or before that period. This has the advantage of combining 'longitudinal' study data and transverse studies.

Pupil diameter: methods of analysis and of presentation

Results were grouped both by postmenstrual age and by the presence or absence of the pupillary light reflex. The correlation coefficient between horizontal and vertical pupil diameters was calculated. Mean pupil diameters were derived from the weekly measurements both for neonates with and without the light reflex. These were then compared using an independent samples $t$ test.

\section{Results}

Fifty premature neonates were studied, approximately balanced for sex. In addition 12 babies born before the onset of the pupil response died before the conclusion of the study and have therefore not been included in the analysis. All neonates were observed initially during their first postnatal week. Those infants who did not have a pupil response at first examination were enrolled in the longitudinal study of pupillary size and reflex development.

The relation between postmenstrual age and the presence of the pupillary light reflex is apparent in fig 1 . In only one baby of less than 30 weeks' postmenstrual age was there a response to light, and in this infant the constriction was both very slow and slight. As there was no pupillary response to light in the next two examinations we concluded that our original observation was incorrect and this result was subsequently excluded from the analysis. No neonate of less than 30 weeks' postmenstrual age had a light reflex; by 34 weeks $86 \%$ (18/21) of all infants examined responded to an increase in illumination with a pupillary constriction and by 35 weeks this light reflex was present in all.

The presence of the pupillary reflex appears to be related to gestational age. The number of neonates who had a pupillary response to light at the initial examination are shown in the table.

When the speed of pupil reaction was taken into account the developmental nature of the light reflex becomes more obvious (fig 2). The changeover from a slow, minimal reaction to the characteristic brisk pupillary response occurred between 31 to 32 weeks' postmenstrual age.

It is of interest to look at the possible effect early exposure may have on the maturation of

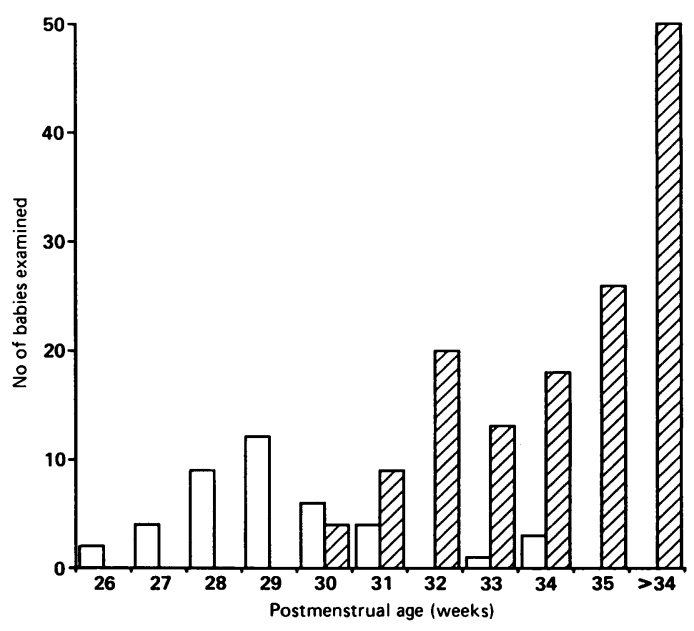

Figure 1 Presence of a pupillary constriction to light for neonates. Each bar represents the number of babies born a that gestation or earlier, with either no reaction $(\square)$ or a pupillary constriction to light $(\mathbb{Z})$. 


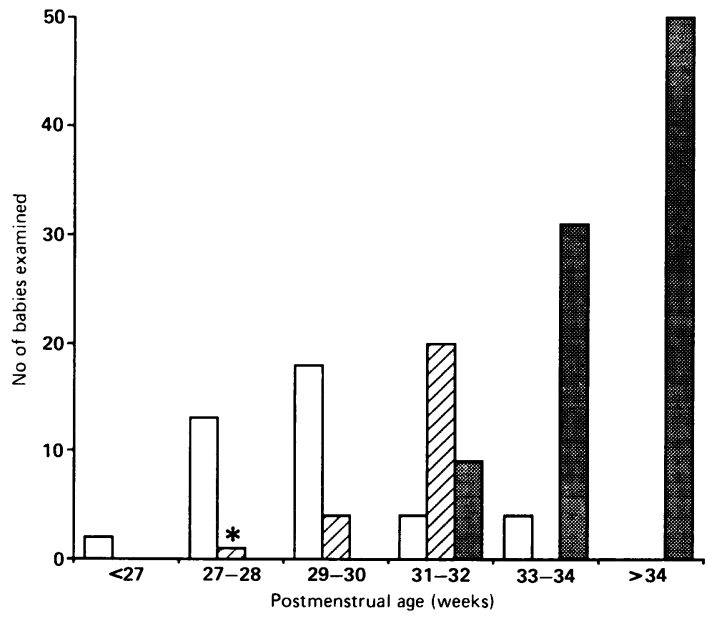

Figure 2 Speeds of pupillary constriction for neonates are grouped by postmenstrual age. Each bar represents the
number of babies examined with no response ( $\square)$; a slow reaction (国) or a normal brisk pupillary constriction to light (口). The asterisk indicates a baby who had a reflex constriction at 27 weeks but was subsequently found to have unreactive pupils when next examined one week and two weeks later.

this reflex. Following infants longitudinally the very immature neonates ( $\leqslant 27$ weeks' gestational age) appeared to develop the pupillary light response at an earlier postmenstrual age than babies born at a later gestation. All babies whose pupils constricted to light at 30 weeks were born at $\leqslant 27$ weeks' gestational age. Thus there is some evidence that early birth hastens the onset of the pupil response but as the number of infants born at this age was small $(n=4)$ this trend could not be examined statistically.

Mean (SD) horizontal and vertical pupil diameters for all infants studied, calculated from the weekly measurements, were $3 \cdot 14$ $(0.07)$ and $3 \cdot 14(0.07)$ respectively. These values include those obtained from infants who had no reflex response to light. As one of the functions of the pupil is to control the amount of light entering the eye it was decided to examine the two subpopulations separately. For babies with no pupillary light reflex (76 weekly measurements) mean horizontal and vertical pupil diameters were $3.46(0.78)$ and $3.49(0.72)$ $\mathrm{mm}$ respectively. While the mean pupil diameters for babies whose pupils reacted to light were $3.02(0.84)$ and $3.00(0.85) \mathrm{mm}$ for the horizontal and vertical diameters respectively ( $n=200$ weekly measurements). When compared using an independent samples $t$ test, infants with no light response were found to have significantly larger pupils than those with a reflex pupillary constriction to light $(p<0.002$ and $\mathrm{p}<0.006$ for the vertical and horizontal diameters respectively). Good interobserver correlation was found $(r=0.93)$.

The colour of the iris was noted as part of the ocular examination. All neonates of less than 34 weeks' postmenstrual age had grey irides, with other colours developing from this time onwards.

\section{Discussion}

This study consists of two major components: first, the development of the pupillary light reflex and second, pupil size under standard neonatal unit illumination (mean 512 lux; range 395-628 lux) before and after the onset of this reflex.

Our observation that the pupillary light reflex was almost invariably absent in neonates of less than 30 weeks differs from Robinson who stated that the pupillary response to light is present in some babies of 29 weeks' gestation. ${ }^{1}$ In the latter study, however, very few neonates of less than 30 weeks' gestational age were examined $(n=7)$ compared with our study $(n=21)$.

The low correlation coefficient between gestation and presence of the pupillary light reflex found by Finnström may also have been due to the small number $(n=6)$ of infants of less than 32 weeks' gestation in the study. ${ }^{2}$

The pathway for the light reflex is subcortical. Thomson suggests that pupillary fibres originate from the retinal photoreceptors, thence to the retinal ganglion cell and the axon of the optic nerve. ${ }^{6}$ These fibres are either very close to or are the same as those involved with visual acuity, as damage to the optic nerve is linked to defects in visual fields with disturbance of pupil responses. They leave the optic tract before the lateral geniculate nucleus to synapse in the pretectal region of the midbrain. Impulses are then relayed by crossed fibres through the posterior commissure to the opposite EdingerWestphal nucleus of the oculomotor nerve. In addition, some fibres pass ventrally to the ipsilateral oculomotor nucleus. The efferent pathway is along the third nerve, via small fibres lying centrally within this nerve, which pass to the ciliary ganglion within the extraocular muscle cone in the orbit. Postganglionic fibres pass via the short ciliary nerves to innervate the sphincter muscle of the iris and other ocular structures.

It is now pertinent to consider some possible mechanism(s) that may account for the onset of the pupillary reflex to light. These include the functional immaturity of the retinal photoreceptors, iris musculature, or neurotransmitters. Other factors to be considered are myelination of the reflex pathway and anatomical restraint provided by the tunica vasculosa lentis. As mentioned the receptors for this response are probably those involved with vision. ${ }^{6}$ That photoreceptor immaturity could explain the absence of the pupil response cannot be excluded. Retinal cones are present by 22 weeks' gestation, ${ }^{7}$ and although they are not morphologically mature for about four years, ${ }^{8}$ it is clearly not possible to correlate accurately morphology and function. It is relevant to note that the blink to light, a reflex dependent on functioning photoreceptors, is present in all babies $\geqslant 26$ weeks' postmenstrual age. ${ }^{1}$

Magoon and Robb have detected traces of myelin in neonates of between 28 and 32 weeks' gestation near the optic chiasm ${ }^{9}$; however, the process of myelination not being completed in the anterior visual pathway for at least two years is probably too slow to account for the rapid onset of the pupil response noted in this study (fig 2). Anatomical restraint by the transient tunica vasculosa lentis and possible immaturity 
of the muscles of the iris need also be considered. It has been suggested that the transient tunica vasculosa lentis may hinder the contraction of the sphincter pupillae muscle thus preventing reflex constriction. ${ }^{1}$ Tunica vasculosa lentis regresses between 28 and 34 weeks' gestation. ${ }^{10}$ However, no anatomical evidence has been found to suggest that this membrane is linked to the iris. Indeed, very immature neonates, born at less than 28 weeks' gestation, are screened before the regression of the transient tunica vasculosa lentis for retinopathy of prematurity and in this situation pupillary pharmacological dilatation can easily be achieved. Finally, the sudden onset of the pupillary reflex could reflect maturation of the iris musculature, either due to insufficient release of or the over efficient reuptake or breakdown of the transmitter (acetylcholine) within the synaptic cleft. Most previous attempts to gain some understanding of the degree of maturity of these muscles in preterm infants have examined the pharmacological effects of various drugs on the muscle dilator pupillae. Lind and associates examined the effects of sympathomimetic amines on the pupil diameter of preterm neonates 28 to 40 weeks' gestational age. ${ }^{11}$ Their results suggested that either nerve endings may not yet be able to release noradrenaline, noradrenaline is unavailable at this age, or that membrane immaturity may prevent uptake of both directly acting amines and of noradrenaline precursors. ${ }^{12}$ They concluded that the end organ is capable of responding but the synthesis storage or release of neurotransmitter may be deficient.

The pupil modifies the amount of light entering the eye. Our results show that neonates who have yet to develop the pupillary light reflex have a larger pupil than those in whom this reflex is established (mean $=3.46 \mathrm{~mm}$ compared with $3.02 \mathrm{~mm}$ ). Only one study has measured pupil size in preterm neonates and this was mainly concerned with the effects of various mydriatic agents on diameter. ${ }^{4}$ These authors measured pupil diameter by comparison with a series of circles of known diameter, obtaining an estimate of $2.9 \mathrm{~mm}$ at 35 weeks' postmenstrual age compared with our results: $3.02 \mathrm{~mm}$ at 35 weeks.

The potentially harmful effects of light on the developing visual system have recently been reviewed. ${ }^{13}$ However, no measurements have been made of the retinal light dose actually received by an infant undergoing treatment on a neonatal unit. Retinal irradiance may be related to pupil diameter, transmission of light across the ocular media, brightness of the room, and the area of the retinal image. ${ }^{3}{ }^{14}$ Hamer and coworkers have made the only previous attempt to calculate the neonatal retinal irradiance that was based on a pupillary diameter of $1.5 \mathrm{~mm},{ }^{3}$ but according to our calculations this needs to be raised by a power of four. Further detailed consideration of this topic is beyond the scope of this paper.

We have shown that the pupil response to light develops between 30 and 31 weeks' postmenstrual age in preterm neonates. The pupil diameter in those infants with no reflex is larger than that in neonates who have developed the light reflex, thus the very immature baby will receive a larger retinal light dose than its older counterpart. We also recorded pupil diameters in a small sample $(n=5)$ of babies whose eyes had been dilated using cyclopentolate $(0.5 \%$ eye drops) before routine screening for retinopathy of prematurity. Mean pupil diameter after dilatation was $5 \cdot 21(0.08) \mathrm{mm}$. Therefore these infants will receive a proportionally greater retinal irradiance until the pharmacologically induced pupillary dilatation wears off.

The consequence of our findings on calculating the neonatal ocular light dose are considerable but further research is needed to determine whether early exposure to light has an adverse or indeed beneficial effect on any part of the immature visual system.

Judith Robinson is a Royal National Institute for the Blind research student. Dr J Thompson provided statistical support.

1 Robinson RJ. Assessment of gestational age by neurological examination. Arch Dis Child 1966;41:437-47.

2 Finnström $\mathrm{O}$. Studies on the maturity in newborn infants. III. Neurological examination. Neuropadiatre 1971;3: 72-96.

3 Hamer RD, Dobson V, Mayer MJ. Absolute thresholds in human infants exposed to continuous illumination. Invest Ophthalmol Vis Sci 1984;25:381-8.

4 Sindel BD, Baker MD, Maisels MJ, Weinstein J. A comparison of the pupillary and cardiovascular effects of various myhydratic agents in preterm infants. $\mathcal{F}$ Pediatr Ophthalmol Strabismus 1986;23:273-7.

5 Alexandridis E. The pupil. (Translated T Telger.) Heidelberg: Springer-Verlag, 1985.

6 Thompson HS. The pupil. In: Moses RA, Hart WM Jr, eds. Adler's clinical physiology of the eye. 8th ed. St Louis: CV Mosby, 1987:311-38.

7 Abramov I, Gordan J, Hendrickson A, Hainline L, Dobson $\mathrm{V}$, la Boissiere $\mathrm{E}$. The retina of the newborn human infant. Science 1985.217.265-7.

8 Hendrickson AE, Yuodelis C. The morphological development of the human fovea. Ophthalmology 1984;91:603-12.

Magoon EH, Robb RM. Development of myelin in human optic nerve and tract. Arch Ophthalmol 1981;98:655-9.

optic nerve and tract. Arch Ophthalmol 1981;98:655-9.
10 Hittner HM, Hirsch NJ, Rudolph AJ. Assessment of gestational age by examination of the anterior capsule of the lens. 7 Pediatr 1977;91:455-8.

11 Lind N, Shinebourne E, Turner P, Cottom D. Adrenergic neurone and receptor activity in the iris of the neonate. Pediatrics 1971;47:105-12.

12 Nicolopoulos D, Agathopoulos A, Galanakos-Tharouniati M, Stergiopolos C. Urinary excretion of catecholamines by fullterm and premature infants. Pediatrics 1969;44:262-7.

13 Moseley MJ, Fielder AR. Light toxicity and the neonatal eye. Clinical Vision Science 1988;3:75-82.

14 Sliney DH. Quantifying retinal irradiance levels in light damage experiments. Curr Eye Res 1984;3:175-9. 